\title{
Outer layer turbulence intensities in smooth- and rough-wall boundary layers
}

\author{
IA N P. CASTRO, ${ }^{1}$ A NTONIO SEGALIN I ${ }^{2}$ \\ and \\ P. HENRIK ALFREDSSON ${ }^{2}$
${ }^{1}$ Aeronautics, Faculty of Engineering and the Environment, University of Southampton SO17 1BJ, UK
${ }^{2}$ Linné Flow Centre, KTH, Stockholm, SE-10044, Sweden

(Received ?? and in revised form ??)

Clear differences in turbulence intensity profiles in smooth, transitional and fully rough zero-pressure gradient boundary layers are demonstrated, using the diagnostic plot introduced by Alfredsson et al. (2011) - $u^{\prime} / U$ vs. $U / U_{e}$ where $u^{\prime}$ and $U$ are the local (rms) fluctuating and mean velocities and $U_{e}$ is the free-stream velocity. A wide range of published data are considered and all zero-pressure gradient boundary layers yield outer flow $u^{\prime} / U$ values which are roughly linearly related to $U / U_{e}$, just as for smooth walls, but with a significantly higher slope which is completely independent of the roughness morphology. The difference in slope is due largely to the influence of the roughness parameter $\left(\Delta U^{+}\right.$ in the usual notation) and all the data can be fitted empirically by using a modified form of the scaling, dependent only on $\Delta U / U_{e}$. The turbulence intensity, at a location in the outer layer where $U / U_{e}$ is fixed, rises monotonically with increasing $\Delta U / U_{e}$ which, however, remains of $\mathcal{O}(1)$ for all possible zero-pressure gradient rough-wall boundary layers even at the highest Reynolds numbers. A measurement of intensity at a point in the outer region of the boundary layer can provide an indication of whether the surface is aerodynamically fully rough, without having to determine the surface stress or effective roughness height. Discussion of the implication for smooth/rough flow universality of differences in outer layer mean velocity wake strength is included.

\section{Background}

There is now a substantial literature on issues concerning universality of smooth- and rough-wall boundary layers, particularly focussed on the question of under what circumstances an aerodynamically rough flow has outer layer characteristics identical to those for a smooth-wall flow, consistent with the original hypothesis of Townsend (1976). For three-dimensional roughness Flack et al. (2005) provide support for this, at least for small roughness height $(k)$ to boundary layer height $(\delta)$ ratios, as do Krogstad \& Efros (2012) in the context of two-dimensional roughness (very small spanwise bars). Recent papers (Castro 2007; Amir \& Castro 2011) present further experimental data, cite much of the surrounding and sometimes contradictory literature but, likewise, conclude that zero-pressure-gradient rough-wall boundary layers are (in the outer flow) indistinguishable from smooth-wall flows, independently of the nature of the roughness but provided $k / \delta$ is sufficiently small. Furthermore, this seems to be valid for surprisingly large $k / \delta$ perhaps up to $15 \%$. Despite this apparent universality, however, there is also considerable evidence that the mean velocity profiles differ in the strength of the wake component. 
The classical profile can be expressed as

$$
U^{+} \equiv \frac{U}{u_{\tau}}=\frac{1}{\kappa} \ln \left(\frac{y u_{\tau}}{\nu}\right)+A-\Delta U^{+}\left(k^{+}\right)+\frac{2 \Pi}{\kappa} w\left(\frac{y}{\delta}\right)
$$

in the usual notation. Note that the $y$-origin is located at the zero-plane displacement height, $d$ (so that the actual height from the bottom of the roughness is $y+d$ ), and the roughness height $k$ is measured from the bottom of the roughness elements to the top of the tallest ones. The roughness function $\Delta U^{+}$is zero for a smooth-wall flow and $\Pi$ is the usual Coles (1956) wake parameter, which is typically about 0.55 for smooth-wall flows provided the Reynolds number is high enough (see, e.g. Nagib \& Chauhan 2008). However, there is little doubt that it can rise to at least 0.7 for fully-rough flows (as found by Krogstad et al. 1992; Bergstrom et al. 2005; Castro 2007, for example).

This difference in wake strength implies that full universality between smooth and rough boundary layers does not exist. But there are major, well-known difficulties in assessing the extent to which the classical two-parameter scaling embodied in the above expression for $U^{+}$accurately describes zero-pressure gradient boundary layers and, if so, over what range of Reynolds numbers. These include the accurate determination of the wall stress and hence $u_{\tau}$, assessing whether spatial resolution of probes is adequate (especially near the wall), and determining the precise probe distance from the wall. The latter two difficulties, in particular, become increasingly serious as the Reynolds number becomes very large (especially for smooth wall flows). In the context largely of smooth-wall flows at high Reynolds numbers, Marusic et al. (2010) have recently explored these issues in examining much of the available data. In commenting on rough-wall flows, they state that the possible interactions between inner and outer regions 'require further investigation' and that, more generally, 'there is a pressing need for further investigation of the rough-wall case'.

Recently, Alfredsson et al. $(2011,2012)$ have introduced what they call the 'diagnostic plot' as a means of assessing, as the Reynolds number increases, the adequacy of scaling laws and of experimental data, including those for the turbulence (at least, the axial intensity). Alfredsson et al.'s diagnostic plot is a convenient way of addressing some of the difficulties mentioned above: $u^{\prime} / U$ (or $u^{\prime} / U_{e}$, where $U_{e}$ is the free-stream velocity) is plotted against $U / U_{e}$ as this avoids use of both $y$ and $u_{\tau}$ and helps to determine whether features identified in the data are genuinely physical. Their interest was restricted to smooth-wall flows and secondary 'outer' peaks in the $u^{\prime+}$ profiles in the near-wall region. However, in view of the even more serious difficulties for rough-wall flows in determining wall stress, wall distance and the zero-plane displacement the approach would seem to have considerable merit for that case too. Initial work on such cases was reported by Segalini et al. (2012); this paper pursues the matter more fully, by re-analysis of many rough-wall data available in the literature. We demonstrate that the diagnostic plot emphasises the clear differences between smooth-wall and fully-rough-wall boundary layers and can provide a rapid assessment of whether or not the flow is transitionally rough. We also discuss the reasons for the high value of wake strength in many rough-wall flows and the implications for true universality.

\section{The diagnostic plot}

Alfredsson et al. $(2011,2012)$ have shown that in a region starting within the logarithmic region and continuing through almost the entire outer wake flow the turbulence intensity $u^{\prime} / U$ decreases linearly with $U / U_{e}$. Also, the extent of this linear region increases with increasing Reynolds number. This is illustrated in figure 1a, for a collection 


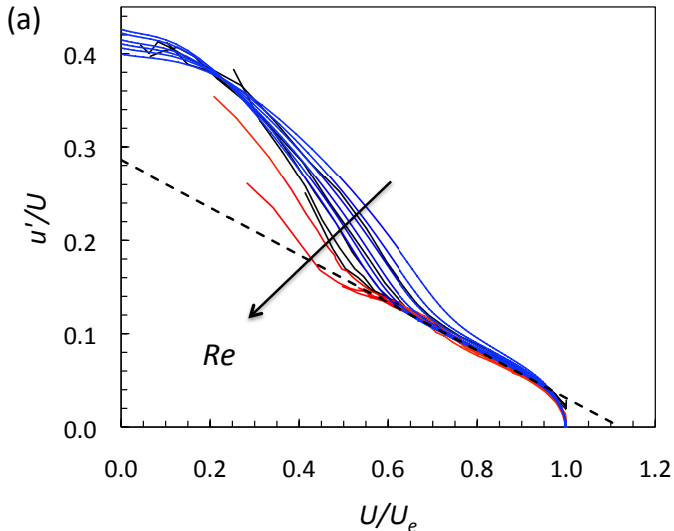

(a)

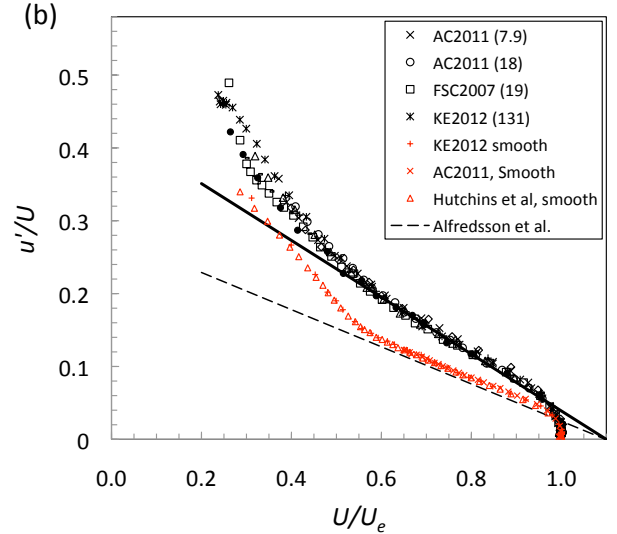

(b)

Figure 1. (a): Turbulence intensity, $u^{\prime} / U$, plotted against the mean velocity for a collection of smooth-wall, zero-pressure-gradient boundary layer data (from Alfredsson et al. 2012). (b) Rough-wall data, from some of the data sets listed in table 1 . The legend includes values of $\delta / k$ for the labelled data; symbols not in the legend are for the Amir mesh surface with smaller $\delta / k$. The solid line provides a reasonable linear fit in the outer layer and the dashed line is the smooth-wall outer layer straight line from Alfredsson et al. (2012).

$\begin{array}{ccccccc}\text { Experiment } & \text { Label } & \text { Roughness } & \delta / k & \delta / k_{s} & \Delta U^{+} & y_{o}^{+} \\ & & & & & \\ \text {Castro \& Snyder (1980) } & \text { FMS, FMF } & \text { Gravel/blocks } & 31,24 & 36,28 & 8.7,11.4 & 4.3,13.5 \\ \text { Snyder \& Castro (2002) } & \text { SC2002 } & \text { Plates } & 59,59 & 36,44 & 8.3,3.4 & 3.7,0.5 \\ \text { Cheng \& Castro (2002) } & \text { CC2002 } & \text { Cubes } & 4.2-10.4 & 3.4-7.2 & 14.1-12.4 & 19.1-41.3 \\ \text { Flack et al. (2005) } & \text { FSC2005 } & \text { mesh } & 19.1-109 & 5.1-55 & 12.6-6.7 & 22.6-1.8 \\ \text { Flack et al. }(2007) & \text { FSC2007 } & \text { Sandgrain } & 54-15.7 & 91-7.0 & 6.0-13.5 & 1.4-24.3 \\ \text { Brzek et al. (2008) } & \text { BCJC08 } & \text { Grit } & 30,86 & 15.7,142 & 9.7,2 & 6.7,0.3 \\ \text { Amir \& Castro (2011) } & \text { AC2011 } & \text { Mesh } & 8.1-29 & 6.0-8.6 & 10.7-13.4 & 9.7-31 \\ \text { Amir \& Castro (2011) } & \text { AC2011 } & \text { Random blocks } & 4.6-18 & 109-311 & 3.6-10.3 & 10.7-17 \\ \text { Amir \& Castro (2011) } & \text { AC2011 } & \text { Grit } & 29-31 & 72-38 & 3.0-8.2 & 0.43-3.5 \\ \text { Volino et al. }(2011) & \text { VSF2011 } & \text { 2D bars } & 32,159 & 2.3,17.7 & 12.7,7.7 & 23.5,3 \\ \text { Volino et al. }(2011) & \text { VSF2011 } & \text { Staggered cubes } & 27.6 & 7.3 & 9.5 & 6.3 \\ \text { Krogstad \& Efros (2012) } & \text { KE2012 } & \text { 2D bars } & 131 & 41 & 13.6 & 36.4\end{array}$

TABLE 1. Details of the experimental data used in the figures. (Note that $\delta / y_{o}$ is $32.6 \delta / k_{s}$. Where two comma-separated entries appear in the $\delta / k$ column, all subsequent pairs in that row have corresponding entries (i.e. in the same order). Where ranges appear, minima and maxima are shown in each column, but the variations for the different parameters are not necessarily monotonic between the limits.)

of smooth-wall zero-pressure-gradient boundary layers. The behaviour conforms to the expected scaling for the axial turbulence, expressed by

$$
\frac{u^{\prime}}{U}=g\left(\frac{U}{U_{e}} ; \frac{U_{e}}{u_{\tau}}\right) .
$$


(a)

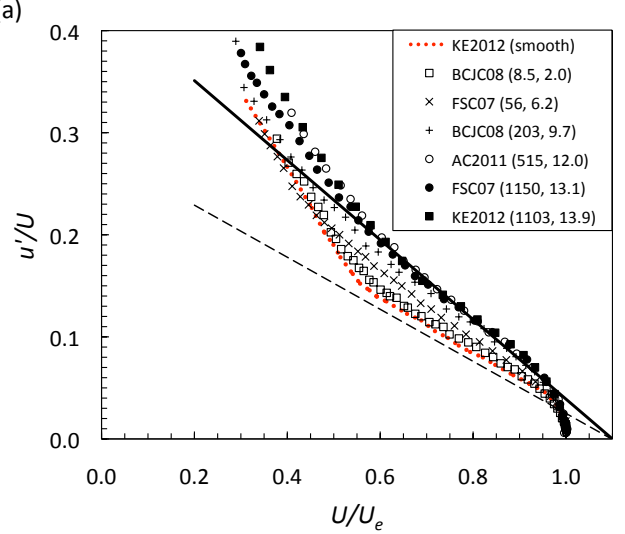

(b)

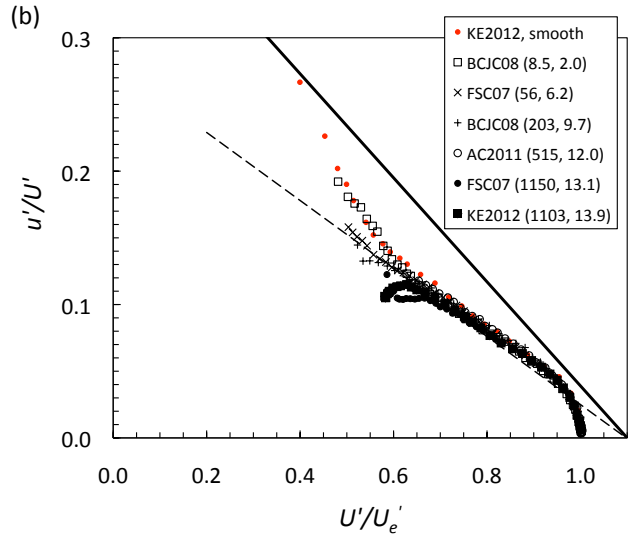

FiguRE 2. (a): Turbulence intensity, $u^{\prime} / U$, plotted against the mean velocity for a sample of rough-wall and transitional boundary layers.(b) The same data as in (a) plotted using $U^{\prime}$ $(U+\Delta U)$ as the scaling velocity. In both figures, the dashed line is the smooth wall line of Alfredsson et al. (2011) and the solid line is the same as in figure 1b. The legend in both plots gives values of $k_{s}^{+}, \Delta U^{+}$for each case.

The linear region is well-fitted by

$$
\frac{u^{\prime}}{U}=a+b \frac{U}{U_{e}}
$$

where $a=0.286 . b=-0.255$ are empirical constants determined from the smooth wall data compilation of Alfredsson et al. (2011). Note that this linear relationship is independent of the Reynolds number (characterised by $U_{e} / u_{\tau}$ in $(2.1)$ ) but becomes more extensive as the Reynolds number rises. Similar collapse to a linear behaviour was shown to occur for (circular) pipe flows and planar (2D) channel flows, although with different values of the slope which, as Alfredsson et al. (2012) pointed out, is probably a result of the different strengths of the wake component (I) in the three flow classes. This would suggest that if rough-wall boundary layers really do have a significantly larger $\Pi$ than that in smooth-wall flows, it would be apparent in the diagnostic plot. Figure 1b shows a selection of fully-rough boundary layer data from various sources and it is immediately clear that the slope of the linear collapse region is indeed significantly larger than it is for smooth-wall flows. Figure 1b also includes smooth-wall data from Hutchins et al. (2011), Amir \& Castro (2011) and Krogstad \& Efros (2012), which collapse in the outer layer and lie close to (although marginally above) the linear smooth-wall line-fit from Alfredsson et al. (2012). (Higher Reynolds number smooth-wall data lie closer to the Alfredsson line.) It is important to emphasise here that although the scaling implied by equation (2.1) arises essentially from a dimensional argument, there is no obvious physical reason for the appearance of a linear region in the outer layer; this seems to be merely a convenient empirical finding.

A significant feature of the data shown in fig.1b is the wide range of $\delta / k$ covered. In particular, Krogstad \& Efros (2012)'s rough-wall data have $\delta / k>100$, which ensures a much more significant separation of inner and outer length scales than is often the case for aerodynamically fully-rough-wall experiments and is perhaps the main reason for expecting universality between smooth and rough cases (Jiménez 2004). Another significant feature is the very different types of roughness morphology the data represent. The surfaces include the expanded mesh of Flack et al. (2005) (with $\operatorname{Re}_{\theta} \approx 13000$ ), the similar but not identical mesh of Amir \& Castro (2011) (for a range of $\delta / k$ and $\operatorname{Re}_{\theta}$ ), the 
array of random-height rectangular blocks of Amir \& Castro $(2011)\left(\operatorname{Re}_{\theta} \approx 12600\right)$ and the two-dimensional spanwise bar surface of Krogstad \& Efros $(2012)\left(\operatorname{Re}_{\theta}=32800\right)$. Other data could have been included. For example, the fully-rough sand-grain surface cases of Flack et al. (2007) and Amir \& Castro (2011). The fact that all these data collapse suggests that for fully rough boundary layers the diagnostic plot is universal in the outer region, independent of roughness morphology. However, the data shown cover flows with roughness functions $\left(\Delta U^{+}\right)$varying between about 9.5 and 14 . A much wider range might not be expected to yield such good collapse (not least because boundary layers with sufficiently low $\Delta U^{+}$are transitionally rough - i.e. viscous effects become non-negligible). This is demonstrated by figure $2 \mathrm{a}$, which shows profiles from a range of flows having increasing values of $k_{s}^{+}$, the traditional 'equivalent sand-grain roughness length', chosen to yield flows with gradually increasing values of $k_{s}^{+}$. Common values of $\kappa$ and $A$ in (1.1)(0.41 and 5.0 respectively) and the classical (Schlichting 1968) empirical relationship for sand-grain roughness, $\Delta U^{+}=\kappa^{-1} \ln k_{s}^{+}+A-8.5$, yield $k_{s}=32.6 y_{o}$ where $y_{o}$ is the roughness length defined by the alternative form of (1.1):

$$
U^{+} \equiv \frac{U}{u_{\tau}}=\frac{1}{\kappa} \ln \left(\frac{y}{y_{o}}\right)+\frac{2 \Pi}{\kappa} w\left(\frac{y}{\delta}\right) .
$$

(1.1) and (2.3) together imply that $\Delta U^{+}=\kappa^{-1} \ln \left(y_{o}^{+}\right)+A ; k_{s}^{+}, y_{o}^{+}$and $\Delta U^{+}$are equivalent ways of characterising the roughness. We prefer the use of $y_{o}^{+}$rather than $k_{s}^{+}$as it is a more direct measure of roughness 'strength' than the rather artificial construct provided by the latter for general roughness morphologies. And $y_{o}^{+}$is certainly more appropriate than $k^{+}$, since $y_{o} / k$ can vary very widely, being highly dependent on roughness morphology. It is clear from figure 2a that as $k_{s}^{+}$rises a roughly linear outer region is still found but the slope of this linear part increases.

Although the differences between smooth- and rough-wall data in figures $1 \mathrm{~b}$ and $2 \mathrm{a}$ might initially be thought to be a consequence solely of wake strength (and can actually be parameterised on that basis alone), this is not so. The full profile model provided by (1.1) with $\Delta U^{+} \neq 0$ properly requires a more appropriate general scaling to that embodied in (2.1), with $U^{+}$being enhanced by the roughness parameter $\Delta U^{+}$(zero for a smooth wall). (2.1) then becomes

$$
\frac{u^{\prime}}{U^{\prime}}=h\left(\frac{U^{\prime}}{U_{e}^{\prime}}\right)
$$

(ignoring possible Reynolds number effects) and, analogously to (2.2), one might anticipate that the data follow

$$
\frac{u^{\prime}}{U^{\prime}}=\tilde{a}+\tilde{b} \frac{U^{\prime}}{U_{e}^{\prime}}
$$

where $U^{\prime}=U+\Delta U$ and $U_{e}^{\prime}=U_{e}+\Delta U$. In the limit of $\Delta U^{+} \rightarrow 0,(2.5)$ will coincide with (2.2) and therefore $\tilde{a}=a$ and $\tilde{b}=b$, where $a$ and $b$ are the diagnostic constants determined from smooth wall data only. Defining the parameter $\Delta U_{n}$ by $\Delta U_{n}=\Delta U^{+} / U_{e}^{+}(2.5)$ can be re-written as

$$
\frac{u^{\prime}}{U}=\frac{U / U_{e}+\Delta U_{n}}{U / U_{e}}\left(a+b \frac{U / U_{e}+\Delta U_{n}}{1+\Delta U_{n}}\right) .
$$

Note that this is not linear in $U / U_{e}$, but the degree of curvature implied by non-zero $\Delta U_{n}$ is small.

The profiles in figure $2 \mathrm{a}$ are re-plotted in figure $2 \mathrm{~b}$ using the scaling implied by (2.4) and it is evident that all the profiles collapse onto the smooth wall result in the outer 

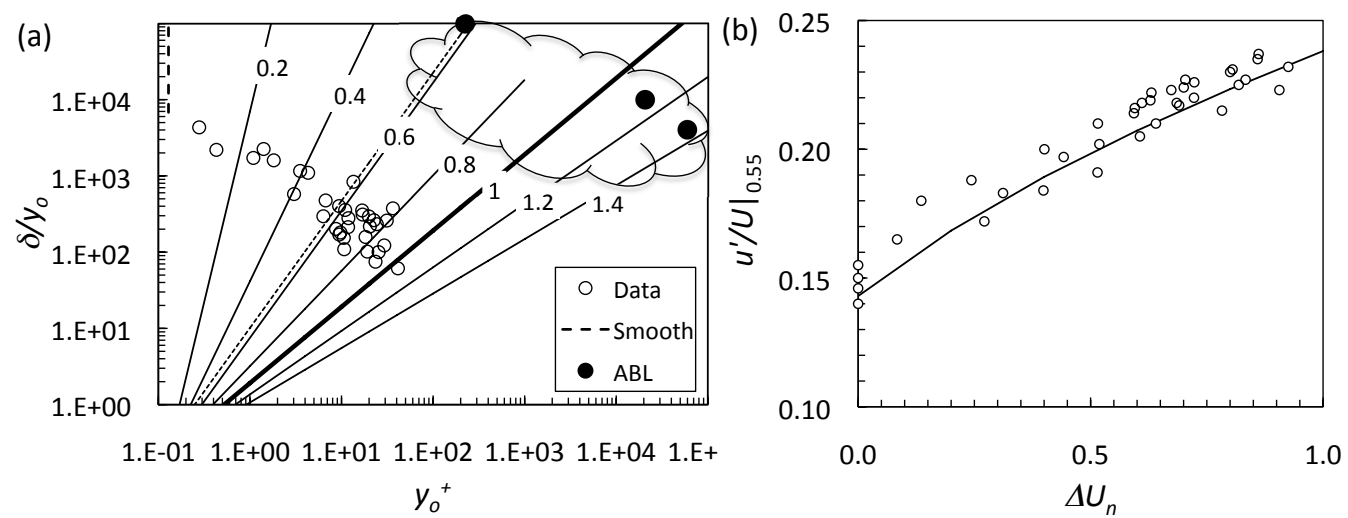

Figure 3. Data for all the cases in previous figures (and given in table 1). (a): values of $\delta^{+} / y_{o}^{+}$ vs. $y_{o}^{+}$. Solid lines (from (2.6)) indicate constant values of $\Delta U_{n}$ (with $\Pi=0.7$ ), ranging from 0.2 to 1.4. Dotted line is for $\Delta U_{n}=0.6, \Pi=0.55$. Large solid symbols (labelled ABL) are typical values for the neutral atmospheric boundary layer, from rural to urban roughness (from Cook 1985) and are surrounded by the 'cloud' to indicate the likely range of all possible ABL data. The vertical dashed line indicates the smooth-wall limit, whose lower bound is chosen to start when $\delta^{+}=1000 ; y_{o}^{+}=e^{-\kappa A}=0.129$ for smooth flows and the upper value of $\delta / y_{o}$ is unbounded. (b): $u^{\prime} / U$ at $U / U_{e}=0.55$ as a function of $\Delta U_{n}$.

layer, independent of $\Delta U^{+}$(or, equivalently, $y_{o}^{+}$). We have not found any data which has a slope greater than that of the solid line in figures $1 \mathrm{~b}$ and 2 and this suggests an upper limit to the right-hand side of (2.6), which would require an upper limit on $\Delta U_{n}$ (since $u^{\prime} / U$ should remain bounded). Now it can be shown that

$$
\Delta U_{n} \equiv \frac{\Delta U^{+}}{U_{e}^{+}}=\frac{\ln \left(y_{o}^{+}\right)+\kappa A}{\ln \left(\delta / y_{o}\right)+2 \Pi},
$$

which does not immediately indicate such a limit. However, on physical grounds the outer layer local turbulence intensities cannot rise without limit as $y_{o}^{+}$increases. Figure $3 \mathrm{a}$ shows contours of constant $\Delta U_{n}$ (with $\Pi=0.7$ ) in the $\delta / y_{o}-y_{o}^{+}$plane along with the data for all the cases in figure $1 \mathrm{~b}$ and figure 2 (and others from the same sources). It is clear that for these laboratory flows $\Delta U_{n}$ hardly exceeds about 1.0. Even in flows with very much larger Reynolds numbers, typified by neutrally stable atmospheric boundary layers (ABL), $\Delta U_{n}$ rarely reaches 1.4 - for extreme winds in very urban (i.e. high roughness length) environments. (The three ABL data points were extracted from compilations in Cook 1985.) Noting that $w(1)=1,(1.1)$ can be used directly to deduce a relation for $\Delta U_{n}, v i z$ :

$$
\Delta U_{n} \equiv \frac{\Delta U^{+}}{U_{e}^{+}}=\sqrt{\frac{C_{f}}{2}}\left(\frac{1}{\kappa} \ln \left(\delta^{+}\right)+B+\frac{2 \Pi}{\kappa}\right)-1,
$$

where $\delta^{+}=\delta u_{\tau} / \nu$ is the Kármán number. Now, the maximum $C_{f}$ in any zero-pressure gradient boundary layer is unlikely to exceed 0.01 at the very most and (2.8) implies that even for such an extreme case $\left.\Delta U_{n}\right|_{\max }$ ranges from about 0.74 to 2.15 for $\delta^{+}$values between $10^{3}$ and $10^{6}$. It seems clear that for fully rough boundary layers $\Delta U_{n}$ must remain an $\mathcal{O}(1)$ quantity whatever the Reynolds number and whatever the roughness.

For all the profiles used to deduce the data in figure $3 \mathrm{a}$, figure $3 \mathrm{~b}$ shows how the turbulence intensity at a fixed location in the boundary layer, chosen (arbitrarily) as the point where $U / U_{e}=0.55$, i.e. usually in the upper half of the log law region for a smooth flow, varies with $\Delta U_{n}$. The data are compared with what would be expected from (2.6) 
- recall that the latter follows (2.5) exactly provided (2.5) yields a reasonable fit to the profile data (as figure $2 \mathrm{~b}$ indicates). There is clearly a monotonic increase in $u^{\prime} /\left.U\right|_{0.55}$ with $\Delta U_{n}$ but, as just argued, we do not expect the latter to much exceed unity even at very high Reynolds numbers - not least because that would imply unphysically large turbulence intensities. Note that alternative choices of the fixed $U / U_{e}$ point (e.g. 0.65) would not alter these conclusions. Note also that cases for which $\Delta U_{n} \lesssim 0.5$ are almost certainly transitionally rough (i.e. $y_{o}^{+}$is not large enough to prevent viscous effects being significant).

\section{The influence of $k / \delta, y_{o}^{+}$and $\delta^{+}$}

The Townsend hypothesis (i.e. that all roughness does is affect the boundary layer thickness and the local skin friction, without altering the structure of the boundary layer in any way) is usually reckoned to be valid provided there is a sufficiently large separation of inner and outer length scales which is often thought to require $k / \delta$ to be sufficiently small. The former can only occur at sufficiently large Reynolds numbers. Jiménez (2004) suggested that $\delta^{+}$needs to exceed about 4000 and that $k / \delta$ should be below $2.5 \%$ at most. The $u^{\prime} / U$ data shown in figure $3 \mathrm{~b}$ are re-plotted in figure $4 \mathrm{a}$ as a function of $k / \delta$ and appear to asymptote to a value around 0.22 but have increasing scatter below about $k / \delta=0.05$. Some data below this value have $u^{\prime} /\left.U\right|_{0.55}$ values similar to those in the large (high roughness) $k / \delta$ range. The clearest example is provided by the Krogstad $\&$ Efros (2012) data, for which $k / \delta$ is only 0.0076 and yet has $u^{\prime} /\left.U\right|_{0.55}=0.225$; this result was anticipated by the entire profile shown in figure $1 \mathrm{~b}$, which collapses very well with the other fully-rough data. There are other data around $k / \delta=0.03$ which have high values of $u^{\prime} /\left.U\right|_{0.55}$. The great scatter in figure 4 a below $k / \delta \approx 0.05$, with data lying between the smooth and the fully rough values, must be largely a result of the variability in $y_{o}^{+}$, rather than specific roughness morphology. If $y_{o}^{+}$is too small viscous effects become significant, although the value of $y_{o}^{+}$at which this happens depends on the specific roughness morphology (Snyder \& Castro 2002). Figure 4b shows the figure 4a plotted as a function of $y_{o}^{+}$demonstrating a reduced scatter and an asymptotic trend towards the fully rough, universal regime at large enough $y_{o}^{+}$. Note that in figure $4 \mathrm{~b}$ the Krogstad \& Efros (2012) data appear at $y_{o}^{+}=36$ - well above the value $y_{o}^{+} \approx 2$ $\left(k_{s}^{+} \approx 70\right)$ generally thought necessary for fully rough conditions. Using this value and Jiménez (2004)'s lower limit criterion for $\delta^{+}(4000)$, the lower limit of $\Delta U_{n}$ (given by (2.7)) for fully rough conditions satisfying Townsend's hypothesis is about 0.30 ; this rises to about 0.37 if one relaxes the scale separation criterion to $\delta^{+}=1000$.

Although $\delta / k$ is the classical parameter used to indicate the degree of scale separation we suggest that, given the significant variation in $y_{o}$ (or, equivalently, $k_{s}$ ) that can arise from different roughness morphologies with the same $k$, a more appropriate measure of scale separation would be provided by $\delta / y_{o}$. It is large enough values of $y_{o}^{+}$and $\delta / y_{o}$ that determines whether the flow can be both fully rough and universal (i.e. the same as a smooth-wall flow in all its essential characteristics). The Krogstad \& Efros (2012) data has $\delta / y_{o}=375$ - more than adequate - whereas some other data, although having values even larger than this, have too small a value of $y_{o}^{+}$. Figure $4 \mathrm{c}$ shows the data plotted as a function of $\delta / y_{0}$. Notice that at large $\delta / y_{0}$ - greater than about 100 , say - values of $u^{\prime} /\left.U\right|_{0.55}$ can nonetheless fall below the fully-rough result, but entirely as a result of too low a value of $y_{o}^{+}$(cf. figure $4 \mathrm{~b}$ ). This emphasises the difficulties in designing appropriate experiments, for too large a value of $\delta / y_{o}$ usually means that the roughness is physically too small to make $y_{o}^{+}$large enough. Unusually long wind tunnels with relatively high free stream speeds are needed to achieve genuinely fully rough (i.e. large enough $y_{o}^{+}$) 

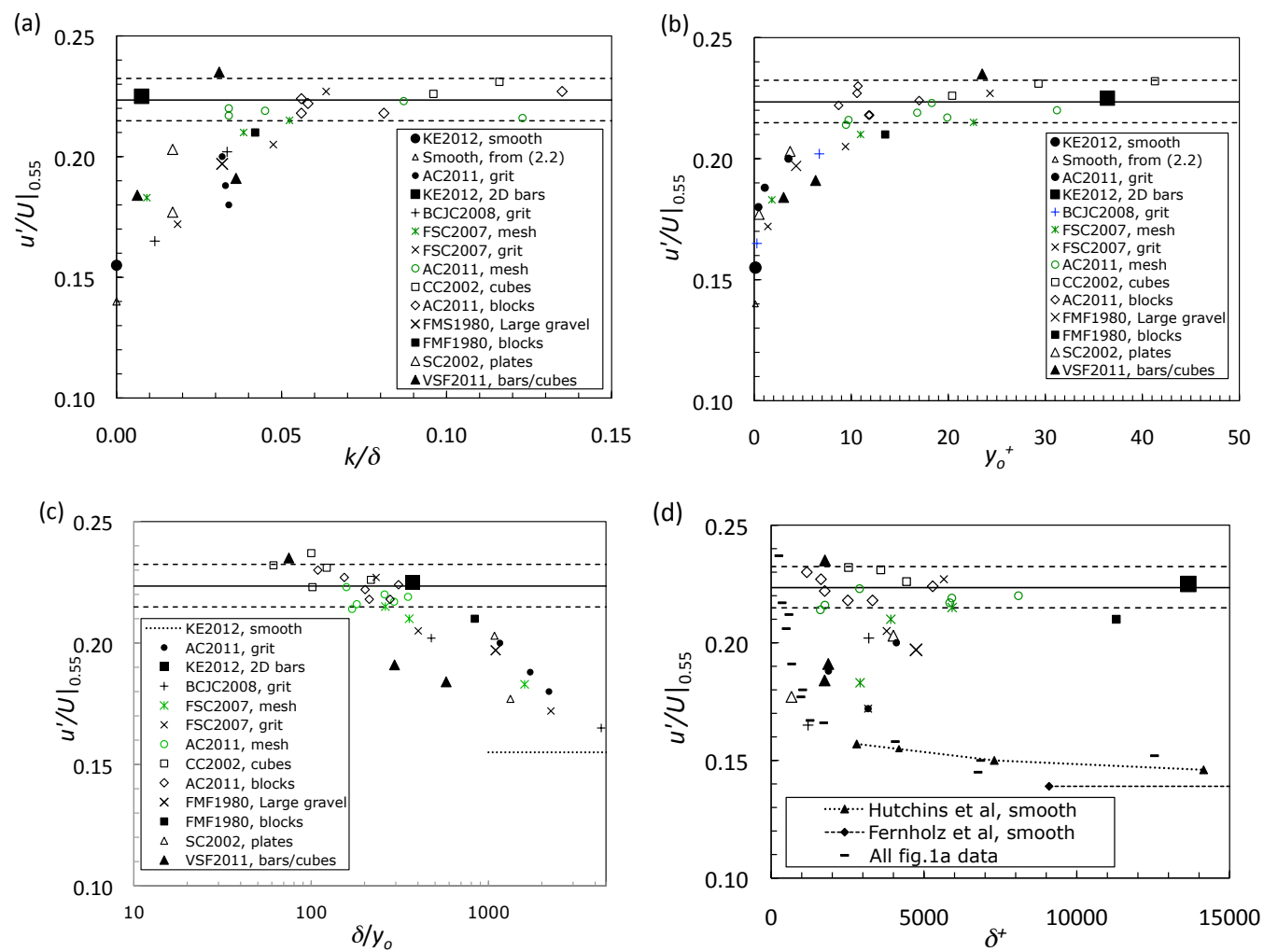

Figure 4. Axial turbulence intensities at the point where $U / U_{e}=0.55$, as a function of (a) $k / \delta$, (b) $y_{o}^{+}$, (c) $\delta / y_{o}$ and (d) $\delta^{+}$. The horizontal solid and dashed lines represent the average for values of $k_{s}^{+}$larger than about 200 and values $\pm 4 \%$ from that average. In (c) and (d), the smooth-wall data (i.e. for $y_{o} \rightarrow 0$ ) is shown by the dotted lines.

boundary layers having $\delta / y_{o}$ in excess of, say, 400. If smaller tunnels are used with a large roughness height, $y_{o}^{+}$might be large enough to ensure fully rough conditions, but $\delta / y_{o}$ might be so small that the flow ceases to have genuine boundary layer characteristics - becoming merely a flow over a set of large obstacles, as Jiménez (2004) argued.

An alternative way of plotting the data is shown in figure $4 \mathrm{~d}$, where the abscissa is the Kármán number, $\delta^{+}$. The figure includes data for a selection of smooth, transitional and fully-rough flows. In this plot, smooth-wall data from the unusually high Reynoldsnumber experiments of Fernholz et al. (1995) and Hutchins et al. (2011) are included; these were obtained in large wind tunnels (in the Netherlands and Australia, respectively). It is clear that even at large $\delta^{+}$, the rough-wall values are significantly higher than the smooth-wall ones (as one expects from (2.6)). Incidentally, the Hutchins et al. (2011) data suggest a slowly falling value with increasing Reynolds number, as implied by the smooth-wall compilation given by Alfredsson et al. (2011) and shown in figure 1a. This would be less noticeable if intensities at, say, $U / U_{e}=0.65$ had been used. Notice also that for the transitionally-rough layers, the rise between smooth and rough values occurs over a range of $\delta^{+}$which depends on the nature of the roughness, just as it does when plotted against the roughness Reynolds number $y_{o}^{+}$(see figure $4 \mathrm{~b}$ ) or the more appropriate scale separation parameter $\delta / y_{o}$ (see figure $4 \mathrm{c}$ ). Even at rather low $\delta^{+}$, a surface may yield high values of $u^{\prime} / U$ in the outer layer if $y_{o}^{+}$and $\delta / y_{o}$ are both sufficiently large. 


\section{Final Discussion}

We emphasise first that any plausible higher value of the wake strength in rough-wall flows $(0.7$, say) than for smooth walls $(0.55)$ does not significantly alter the relation between the scale ratio, $\delta / y_{o}$, and $y_{o}^{+}$at fixed $\Delta U_{n} \equiv \Delta U^{+} / U_{e}^{+}$. This is demonstrated in figure 3a where, as an example, the implication of (2.7) for the $\Delta U_{n}=0.6$ contour but with $\Pi=0.55$ rather than 0.7 is included. However, the fact that the wake strength seems to be different for rough-wall flows, increasing gradually with $\Delta U^{+}$(e.g. Bergstrom et al. 2005; Castro 2007) whilst not affecting the collapse of smooth- and rough-wall Reynolds stress profiles, would appear to indicate a lack of complete universality between smoothand rough-wall flows. Figure 5b confirms again the outer-layer collapse of Reynolds shear stresses for smooth and rough walls. When plotted against $U / U_{e}$, rather than $U^{\prime} / U_{e}^{\prime}$, smooth and rough-wall profiles separate, as anticipated - from figure $1 \mathrm{~b}$, for example and as shown in figure $5 \mathrm{a}$. Since it is only the turbulent shear stress which appears in the (first-order) mean momentum equation and can thus affect the mean velocity profile, one is prompted to ask why the wake strength should be different. Might it not simply be a result of too low a value of $\delta / y_{0}$ to ensure sufficient scale separation, or is it because, despite collapse of all second moments, the turbulence structure is rather different?

There are many suggestions in the literature about why the turbulence structure in the outer layer of a rough-wall flow may differ from that over smooth walls. One of the first was that of Grass (1971) who argued that entrainment near rough surfaces is much more violent than near smooth surfaces, associated with important differences in the ejection-sweep cycles. Likewise, Antonia (1971) found that energy transfer via the triple velocity products is not a gradient diffusion process near a rough surface, as later emphasised by Andreopoulos \& Bradshaw (1981) who concluded that turbulence energy sinks may exist near roughness elements. The latter authors found significantly higher triple product levels in rough- than in smooth-wall flows. Orlandi \& Leonardi (2008) have argued that it is the vertical component of the fluctuation velocity which may provide a better indication (than, say, $y_{o}^{+}$) of the roughness effects. Data from the experiments cited here certainly show that $v^{\prime} / U$ (at fixed $y / \delta$ ) rises monotonically with $k / \delta$, as previously discussed by Orlandi \& Leonardi (2008) and Amir \& Castro (2011). Furthermore, there have been a number of studies of the details of the turbulence structure (e.g. Flack et al. 2005, 2007; Krogstad \& Antonia 1999; Krogstad \& Efros 2012), some of which provide conflicting evidence concerning structural characteristics in the outer layer and the extent to which they may be influenced by the inevitable changes in the inner layer caused by roughness.

The recent flow described by Krogstad \& Efros (2012) is again particularly significant, however, since this is one of the few cases which has a fully rough surface $\left(y_{o}^{+}=36\right)$, a high Reynolds number $\left(\delta^{+}=13,650\right)$ and a high $\delta / y_{o}(375)$, guaranteeing adequate scale separation. This is probably because this case used two-dimensional bar roughness, which is known to give a significantly larger $y_{o} / k$ (or, equivalently, $k_{s} / k$ ) ratio than most three-dimensional roughness morphologies. Their study demonstrated that the detailed turbulence structure (triple velocity products, pdfs, quadrant analysis data, etc.) was in fact the same as in a high Reynolds number smooth-wall flow. In particular, in the current context, the outer layer wake strength was only around 0.51 - typical of smoothwall flows. On the other hand, most of the other fully rough $\left(y_{o}^{+}>\approx 2\right)$ data discussed here are from cases which had too small a value of $\delta / y_{o}$ to ensure adequate scale separation and this presumably allowed the altered near-surface flow to affect the outer flow at least to the extent of altering the wake strength, even though outer layer stress profiles collapse closely with those over smooth walls. This would also explain the noticeable changes that 

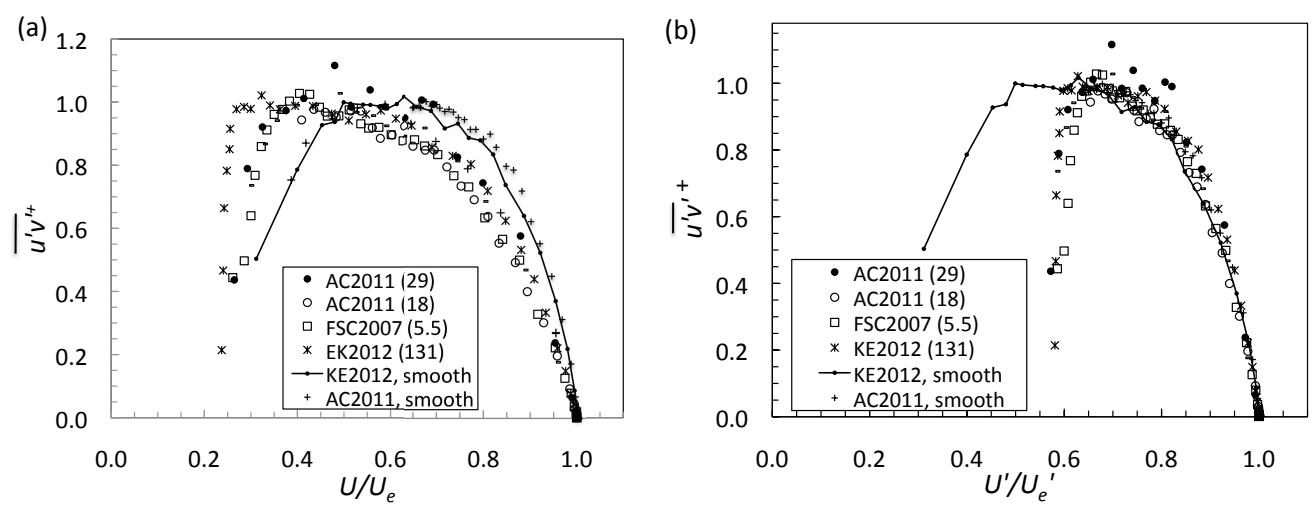

FiguRE 5. Reynolds shear stress profiles for some of the cases used in previous figures, plotted against (a) $U / U_{e}$, (b) $U^{\prime} / U_{e}^{\prime}$.

occur in turbulence structure in the outer flow. Recognising that most of the cases in figure 4 having $\delta / y_{o} \lesssim 300$ had wake strengths rather higher than the smooth-wall value, we suggest that this value might provide a reasonable criterion to ensure sufficient scale separation, with $y_{o} \gtrsim 10$ ensuring (for many morphologies) fully rough conditions, so that if both these are satisfied the boundary layer structure may be properly universal in all respects. Note, however, that for some roughness morphologies rather smaller $y_{o}^{+}$ may be sufficient to ensure fully rough conditions.

In any case, the results presented here demonstrate that despite the different nature of smooth, transitional and fully-rough boundary layers in the near-surface region, outer layer turbulence intensities, when presented in the form of the diagnostic plot introduced by Alfredsson et al. (2011) but re-scaled to account for the roughness function, collapse (figure 2b). There is similar outer-layer collapse in profiles of the normal component of the fluctuating velocity (not shown) and, as noted above, the shear stress. Incidentally, the fall in shear stress below $U^{\prime} / U_{e}^{\prime} \approx 0.62$, evident in figure $5 \mathrm{~b}$, is not a result of increasing viscous effects, as in the smooth-wall case, but simply because this marks the approximate top of the roughness sub-layer (defined as the region in which flow variables are inhomogeneous in horizontal planes). Below this height, $y_{\text {top RS }}$ say, Reynolds stresses (and mean velocity) depend on spatial location.

We make a final comment concerning the depth of this roughness sublayer which is known to vary widely for different roughness morphologies. By assuming that the location where $U^{\prime} / U_{e}^{\prime} \approx 0.62$ corresponds to the top of the roughness sublayer, the measured mean velocity at the same location will be $U / U_{e} \approx 0.62-0.38 \Delta U_{n}$. Equation (2.3) then implies that the associated $y$ position is

$$
y_{\text {top RS }}=y_{o} \exp \left[\kappa U_{e}^{+}\left(0.62-0.38 \Delta U_{n}\right)\right] .
$$

Consider as examples the Krogstad \& Efros (2012) case of 2D bars and the Flack et al. (2007) case having the largest mesh. The respective values of the $\left(U_{e}^{+}, \Delta U_{n}\right)$ pair for these cases, $(16.9,0.819)$ and $(16.1,0.783)$, yield (using the appropriate $\left.y_{o} / k\right) y_{\text {top Rs }} / k=3.4$ and 0.7 . The corresponding measured values, taken as the location where the shear stress begins to fall rapidly, are 2.3 and 1.05 , respectively. Whilst the calculated values are not too far from those measured, $y_{\text {top RS }}<k$ (as in the second case) is not physically realistic and is a result of too small a value of $U^{\prime} / U_{e}^{\prime}$ used in (4.1), compared with that measured (0.646). $y_{\text {top RS }}$ is very sensitive to the value of $U^{\prime} / U_{e}^{\prime}$ chosen and it is clear that (4.1) is not a very precise way of estimating the depth of the roughness sublayer. 
Overall, we conclude that, following the spirit of Alfredsson et al. (2011) and plotting mean flow profiles in a way which avoids the uncertainties associated with determining surface stress, wall distance and roughness function (whose accurate determination is equally problematic), data plotted simply as $u^{\prime} / U$ vs. $U / U_{e}$ - the most easily measurable quantities - do not collapse (figure 2a). This is a result predominantly of the non-zero roughness function $\Delta U^{+}$rather than any differences in outer layer wake strength which, if present, are probably caused by inadequate scale separation. However, data for fully rough situations (with $\Delta U^{+} \gtrsim 7$ ) do collapse quite closely among themselves, as seen in figure $1 \mathrm{~b}$, despite the very different surface morphologies in all these cases. Given the continual rise in turbulence intensity with increasing $\Delta U / U_{e}$ at any arbitrary point in the outer region (but at the same $U / U_{e}$ ), see (2.6) and figure $3 \mathrm{~b}$, for example, it is not in principle possible to use the measured intensity at such a point as a test for whether the flow is transitionally or fully rough. Nevertheless, laboratory flows will almost never have $\Delta U^{+}>14$ even at the highest Reynolds number $\left(\delta^{+}\right)$and a measurement of $u^{\prime} / U$ at a fixed value of $U / U_{e}(0.55$, say) in the outer layer provides a rapid pragmatic assessment of whether the flow is fully rough or not, since for transitional cases its value would lie between the two limiting straight lines shown in figure $1 \mathrm{~b}$.

\section{Acknowledgements}

We are grateful to all those who supplied their original data - Profs. Castillo (via Dr Brzek), Flack, Ganapathisubramani and Krogstad. Other data used were available in our own archives or deduced from published papers. Helpful discussions with our colleagues Profs Ganapathisubramani, Coleman and Örlü were also invaluable, as were comments by the referees.

\section{REFERENCES}

Alfredsson, P.H., Örlü, R \& Segalini, A. 2012 A new formulation for the streamwise turbulence intensity distribution in wall-bounded turbulent flows. Eur. J. Mech $/ B \mathbf{3 6}$, $167-175$.

Alfredsson, P.H., Segalini, A. \& ÖRLü, R. 2011 A new scaling for the streamwise turbulence intensity in wall-bounded turbulent flows and what it tells us about the outer peak. Phys. Fluids 23, 041702.

Amir, M. \& Castro, I. P. 2011 Turbulence in rough-wall boundary layers: universality issues. Exp. Fluids 51, 313-326.

Andreopoulos, J. \& Bradshaw, P. 1981 Measurements of turbulence structure in the boundary layer on a rough surface. Boundary Layer Met. 20, 201-213.

Antonia, R.A. ABD Luxton, R.E. 1971 Energy balance in a turbulent boundary layer on a rough wall. Phys. Fluids 14, 1027-1029.

Bergstrom, D.J., Akinlade, O.G. \& Tachie, M.F. 2005 Skin friction correlation for smooth and rough wall turbulent boundary layers. ASME J Fluids Engng 127, 1146-1153.

Brzek, BG, Cal, RB, Johansson, G \& Castillo, L 2008 Transitionally rough zero pressure gradient turbulent boundary layers. Exp. Fluids 44, 115-124.

CAstro, I.P. 2007 Rough-wall boundary layers: mean flow universality. J. Fluid Mech. 585, $469-485$.

CAstro, I.P. \& SNyder, W.H. 1980 Three naturally grown and simulated boundary layers. Fluid Modelling Facility report, US Environmental Protection Agency. Unpublished report.

Cheng, H. \& Castro, I.P. 2002 Near-wall flow over urban-type roughness. Boundary Layer Met. 104, 229-259.

Coles, D.E. 1956 The law of the wake in a turbulent boundary layer. J. Fluid Mech. 1, 191-226.

Cook, N. 1985 Designers Guide to Wind Loading on Building Structures: Part 1. Butterworths.

Fernholz, H.H., Krause, E., Nockerman, M. \& Schober, M 1995 Comparative measure- 
ments in the canonical boundary layer at $R e_{\delta_{2}}<60000$ on the wall of the German-Dutch wind tunnel. Phys. Fluids 7, 1275-1281.

Flack, K.A., Schultz, M.P. \& Connelly, J.S. 2007 Examination of a critical roughness height for outer layer similarity. Phys. Fluids 19, 095104.

Flack, K.A., Schultz, M.P. \& Shapiro, T.A. 2005 Experimental support for Townsend's Reynolds number similarity hypothesis on rough walls. Phys. Fluids 17, 035102.

Grass, A.J. 1971 Structural features of turbulent flow over smooth and rough boundaries. J. Fluid Mech. 50, 233-255.

Hutchins, N., Monty, J.P., Ganapathisubramani, B., NG, H.C.G. \& Marusic, I. 2011 Three-dimensional conditional structure of a high-Reynolds-number turbulent boundary layer. J. Fluid Mech. 673, 255-285.

JimÉnez, J. 2004 Turbulent flow over rough walls. Annu. Rev. Fluid Mech. 36, 173-196.

Krogstad, P.-Ȧ. \& Antonia, R. 1999 Surface roughness effects in turbulent boundary layers. Exp. Fluids 27, 450-460.

Krogstad, P.-Ȧ., Antonia, R.A. \& Browne, L.W.B. 1992 Comparison between rough- and smooth-wall turbulent boundary layers. J. Fluid Mech. 245, 599-617.

Krogstad, P.-A. \& Efros, V. 2012 About turbulence statistics in the outer part of a boundary layer developing over 2D surface roughness. Phys. Fluids 24, 075112.

Marusic, I., McKeon, B.J., Monkewitz, P.A., Nagib, H.M.N., Smits, A.J. \& K.R., SREenivasan 2010 Wall-bounded turbulent flows at high Reynolds numbers: recent advances and key issues. Phys. Fluids 22, 065103.

Nagib, H.M. \& Chauhan, K.A. 2008 Variations of von Kármán coefficient in canonical flows. Phys. Fluids 20, 101518.

Orlandi, P. \& LeONARDi, S. 2008 Direct numerical simulation of three-dimensional turbulent rough channels: parameterization and flow physics. J. Fluid Mech. 606, 399-415.

Schlichting, H. 1968 Boundary layer theory, 6th edn. McGraw-Hill.

Segalini, A., Örlü, Castro, I.P. \& \& Alfredsson, P.H. 2012 The streamwise turbulence internsity - a comparison between smooth and rough wall turbulent boundary layers. In Prog. in Turbulence $V$. iTi Conference on Turbulence V.

SNyder, W.H. \& CASTRO, I.P. 2002 The critical Reynolds number for rough-wall boundary layers. J. Wind Eng. Ind. Aero. 90, 41-54.

Townsend, A.A. 1976 The structure of turbulent shear flow, 2nd edn. CUP.

Volino, RJ, Schultz, MP \& Flack, KA 2011 Turbulence structure in boundary layers over periodic two- and three-dimensional roughness. J. Fluid Mech. 676, 172-190. 